\title{
Response to Afonso et al.
}

\author{
Benoît Pilmis ${ }^{1}$ • J.-R. Zahar ${ }^{1}$
}

Received: 27 July 2020 / Revised: 27 July 2020 / Accepted: 27 July 2020 / Published online: 5 August 2020

(C) Springer-Verlag GmbH Germany, part of Springer Nature 2020

\section{Dear Editor,}

We read with great interest the correspondence by Afonso et al. highlighting the difference between overall mortality and attributable mortality related to Pseudomonas aeruginosa infections. First of all, we would like to thank them for their remark allowing us to clarify our message. As suggested, additional analyses were conducted to determine the mortality rate attributable to Pseudomonas aeruginosa infection in our population. Also, we defined the attributable mortality related to Pseudomonas aeruginosa if children died while blood culture still positive or if the death occurs within the first $72 \mathrm{~h}$ after the start of antibiotic therapy. According to this definition, the attributable mortality rate was $11 \%(n=13)$, in our study comparable to the mortality observed by Blot et al. [1]. Among these thirteen children, only six were treated with combination therapy. We would like to highlight that most of the children included in our study had severe co-morbidities, including 62 immunosuppressed patients and 21 patients with congenital heart diseases, explaining the high overall mortality rate (31.4\%).

Authors' contributions Benoît Pilmis: Writting JR Zahar: Reviewing

\section{Reference}

1. Blot S, Vandewoude K, Hoste E, Colardyn F (2003) Reappraisal of attributable mortality in critically ill patients with nosocomial bacteraemia involving Pseudomonas aeruginosa. J Hosp Infect 53: 18-24. https://doi.org/10.1053/jhin.2002.1329

Publisher's note Springer Nature remains neutral with regard to jurisdictional claims in published maps and institutional affiliations.

Communicated by Peter de winter

Benoît Pilmis

bpilmis@gmail.com

1 Hopital Universitaire Necker-Enfants Malades, Paris, France 